\title{
Fatiga cíclica en sistemas reciprocantes WaveOne Gold y Reciproc Blue después de su uso en canales artificiales con curvaturas severas Cyclical fatigue in reciprocating WaveOne Gold and Reciproc Blue systems after use in severely curved artificial canals
}

\author{
Angel Lara ${ }^{1}$ \\ Daniela Mendoza ${ }^{2}$
}

'Universidad San Francisco de Quito, Colegio de Ciencias de la Salud, Escuela de Odontología, Clínica de Odontología, Campus Cumbayá, oficina C0 106, casilla postal 17200-841. Quito-Ecuador. Correo electrónico: ange-ger2310@hotmail.com ${ }^{2}$ Universidad San Francisco de Quito, Colegio de Ciencias de la Salud, Escuela de Odontología, Clínica de Odontologia, Campus Cumbayá, oficina C0 106, casilla postal 17200-841. Quito-Ecuador. Correo electrónico: madanimendoza@gmail.com

Editado por / Edited by: Johanna Monar

Recibido / Received: 9-12-2020

Aceptado / Accepted: 23-02-2021 


\section{Resumen}

El objetivo del presente estudio fue comparar el número de ciclos hasta la fractura (NCF) de dos limas endodónticas reciprocantes, la WaveOne Gold Primary (WOGP) y la Reciproc Blue R25 (RBR25) después de instrumentar canales artificiales de acero inoxidable con ángulo de curvaturas de $60^{\circ}$ y radios de curvatura de 20 $5 \mathrm{~mm}$. Se formaron cuatro grupos de estudio: $1^{\circ}$ WOGP con $2 \mathrm{~mm}$ de radio, $2^{\circ}$ WOGP con $5 \mathrm{~mm}$ de radio, $3^{\circ} \mathrm{RBR} 25 \mathrm{con} 2 \mathrm{~mm}$ de radio y $4^{\circ} \mathrm{RBR} 25 \mathrm{con} 5 \mathrm{~mm}$ de radio; de 5 instrumentos cada uno. Se tomó el tiempo de instrumentación en segundos hasta la fractura y así calcular el NCF. El grupo 4 tuvo mayor NCF seguido del grupo 3, del grupo 2 y del grupo 1. Ambos grupos Reciproc R25 fueron significativamente superiores en la resistencia a la fatiga cíclica que los grupos Wave One Gold Primary. Posterior a la fractura se observaron las zonas separadas transversales con microscopio de Endodoncia.

Palabras clave: número de ciclos, fatiga cíclica, fractura, WaveOne Gold, Reciproc Blue

\section{Abstract}

The aim of this study was to compare the number of cycles to breakage (NCF) of WaveOne Gold Primary (WOGP) and Reciproc Blue R25 (RBR25), after their use on root canals with $60^{\circ}$ of curvature and radius ( 2 or $5 \mathrm{~mm}$ ). Therefore, four groups: $1^{\circ}$ WOGP with a $2 \mathrm{~mm}$. radius; $2^{\circ}$ WOGP with a $5 \mathrm{~mm}$. radius; $3^{\circ} \mathrm{RBR} 25$ with a $2 \mathrm{~mm}$. radius; and $4^{\circ} \mathrm{RBR} 25$ with a $5 \mathrm{~mm}$. radius. of 5 files. Root canals were shaped with $60^{\circ}$ and with a $2 \mathrm{~mm}$. and $5 \mathrm{~mm}$. radius, and time until file breakage was recorded and this is how the NCF was obtained. Cross section after file breakage was observed with an Endodontic Microscope. Group 4 had higher NCF, followed by group 3, group 2 and group 1. Both groups RBR25 had significant better cyclic fatigue resistance than WOGP.

Keywords: number of cycles, cyclic fatigue, fracture, WaveOne Gold, Reciproc Blue. 


\section{Introducción}

La Endodoncia es una rama especializada de la odontología que estudia los tejidos del interior de los dientes y su relación con los perirradiculares; con el fin de tratar patologías en ellos, mantener el diente en función y lograr reparación periodontal. Para cumplir estos objetivos se requiere eliminar el tejido pulpar inflamado e infectado por presencia microbiana.

La instrumentación de los conductos radiculares es una de las bases del tratamiento endodóntico estableciendo una forma cónica que hace accesible la irrigación y obturación de los mismos. Existen muchas técnicas e instrumentos para la conformación de los conductos que han ido perfeccionando a través del tiempo de la mano de avances tecnológicos en cuanto al diseño, la aleación y del tipo de movimiento de las limas endodónticas.

La cinemática de instrumentación más usada en la actualidad es la Reciprocante, pues combina movimientos con giros horarios y antihorarios en diferentes grados reduciendo el índice de fractura de los instrumentos, para ello se usan limas rotatorias con aleaciones muy flexibles termotratadas. Los sistemas reciprocantes como WaveOne Gold y Reciproc Blue son elaborados con tratamiento térmico del Níquel-Titanio patentados por sus respectivas casas comerciales, y son las más usadas en la actualidad por presentar en varios estudios previos menor riesgo de fractura en relación a otros sistemas. Sin embargo, a pesar de las ventajas de la técnica como una mayor rapidez, buena eficacia de corte y adecuada capacidad de mantenerse centrado en el canal, no existe un sistema mecanizado infalible que evite la fractura, por lo que el riesgo de fatiga cíclica que conlleva a la fractura sigue siendo la principal desventaja de su uso.

La separación de un instrumento altera el pronóstico del tratamiento, debido a que el fragmento roto impide la completa desinfección microbiana en el sitio ocupado por el segmento fracturado. Algunos métodos para prevenir la separación de una lima son la evaluación visual y microscópica de los defectos superficiales que podamos encontrar en la lima después de utilizarla, o llevar un control estricto del número de ciclos según el tiempo para no sobre-exponerlo a la fatiga. Y ha sido interés del presente estudio analizar un indicativo que puede prevenir la fractura como el número de ciclos hasta la fractura (NCF) en dos tipos de limas reciprocantes como son WaveOne Gold Primary y Reciproc Blue R25 después de la instrumentación de canales artificiales de acero inoxidable con curvaturas severas. Teniendo como hipótesis que el instrumento de Endodoncia Reciproc Blue R25 tiene mayor número de ciclos hasta la fractura después de su uso en canales con curvatura severa que el instrumento WaveOne Gold Primary.

\section{Metodología}

La investigación es de tipo experimental, in vitro, comparativo y descriptivo. La muestra consiste en 20 limas endodónticas reciprocantes de similares caracte- 
rísticas, 10 Reciproc Blue R25 y 10 WaveOne Gold Primary; que se utilizaron para instrumentar canales artificiales con curvatura severas según la clasificación de Schneider de ángulo de curvatura de $60^{\circ}$ y 2 tipos de radios de curvatura de $2 \mathrm{~mm}$ y de $5 \mathrm{~mm}$, fabricados en acero inoxidable por industria metalúrgica ecuatoriana. ${ }^{1}$

Se dividieron 4 grupos de estudio denominados con los números del 1 al 4, con 5 limas reciprocantes nuevas y estériles de $25 \mathrm{~mm}$ de largo cada uno de ellos, como se detalla en la Tabla \#1.

Tabla \#1: Clasificación de los grupos de estudio.

\begin{tabular}{|c|c|c|c|}
\hline $\begin{array}{l}\text { Denominación } \\
\text { del Grupo }\end{array}$ & $\begin{array}{l}\mathrm{N}^{\circ} \mathrm{de} \\
\text { limas }\end{array}$ & Tipo de lima & $\begin{array}{l}\text { Canal con curvatura severa } \\
\text { por tipo de angulo y radio }\end{array}$ \\
\hline 1 & 5 & $\begin{array}{l}\text { WaveOne Gold (WOG) } \\
\text { Primary }\end{array}$ & $60^{\circ}$ de ángulo y $2 \mathrm{~mm}$ de radio \\
\hline 2 & 5 & $\begin{array}{l}\text { WaveOne Gold (WOG) } \\
\text { Primary }\end{array}$ & $60^{\circ}$ de ángulo y $5 \mathrm{~mm}$ de radio \\
\hline 3 & 5 & Reciproc Blue R25 & $60^{\circ}$ de ángulo y $2 \mathrm{~mm}$ de radio \\
\hline 4 & 5 & Reciproc Blue R25 & $60^{\circ}$ de ángulo y $5 \mathrm{~mm}$ de radio \\
\hline
\end{tabular}

Se fabricó un soporte con un diseño propio del autor para mantener la pieza de mano del motor de endodoncia X Smart Plus (Dentsply Maillefer) en un mismo sitio de tal manera que la instrumentación de los conductos sea precisa, estable y estandarizada a la misma longitud de trabajo en todas las muestras del estudio. Este diseño se logró con un articulador dental de tipo Annhua (Modelo 8542026071) modificado con masillas y abrazaderas plásticas colocadas en puntos específicos y adaptadas para formar una sola estructura, que permiten a la pieza de mano maniobrarla con movimientos de intrusión y extrusión como es la indicación para el uso de limas endodónticas. Además, en la base del soporte articulado se talló un rectángulo del mismo tamaño de las muflas de acero con el fin de posicionarlas establemente para que la lima reciprocante coincida con el canal a ser instrumentado. 


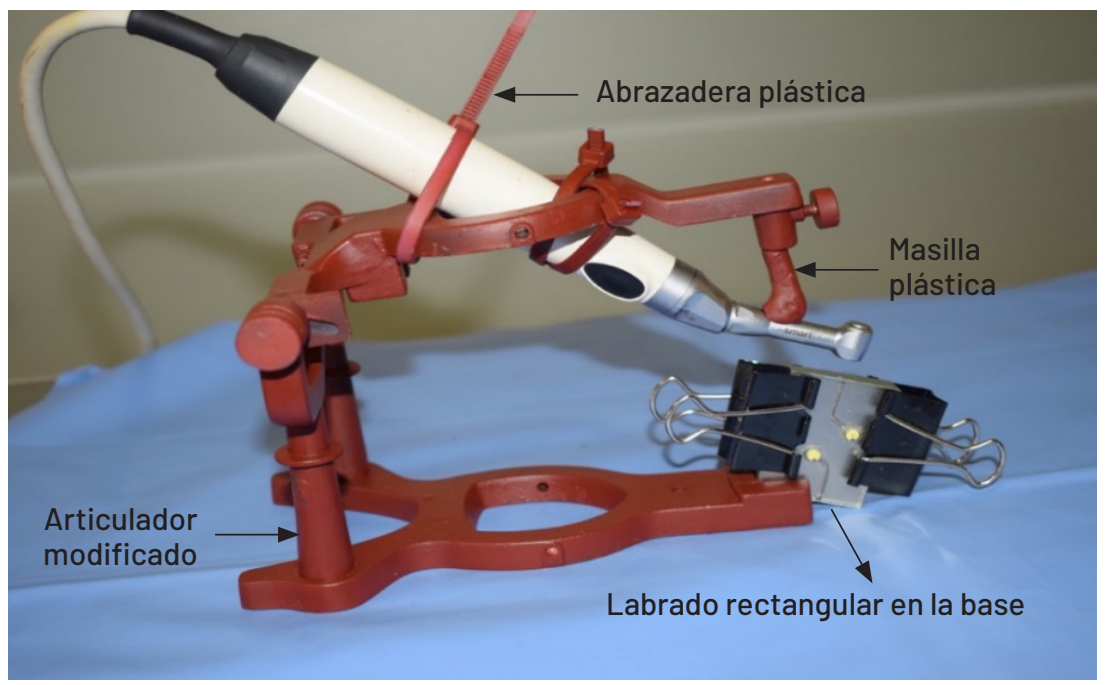

Figura \#1. Soporte para pieza de mano.

Se confeccionaron 4 bloques acero inoxidable con labrados internos de 2 tipos de canales con curvas severas en cada uno, dando un total de 8 conductos de 60은 de ángulo de curvatura, 4 de ellos con radio de $2 \mathrm{~mm}$ y otros 4 con radio de $5 \mathrm{~mm}$. Cada conducto artificial con $17 \mathrm{~mm}$ de longitud, una conicidad variable de 0,0313 en cada $\mathrm{mm}$, un orificio de entrada de $1.5 \mathrm{~mm}$, un orificio final de $1 \mathrm{~mm}$ de ancho y una zona circular final al canal de $4 \mathrm{~mm}$ de diámetro para el ingreso del irrigante. Para conformar muflas de estudio con conductos similares a los dentarios, se fijaron placas de vidrio desmontables y transparentes, de iguales medidas a los bloques de acero, sobre estos con pinzas papeleras Smartgo; pero las placas fueron previamente perforadas a la altura del ápice de los canales y cubiertas con topes de goma con el fin de aplicar hipoclorito de sodio al 5,25\% en jeringa por el ápice para permtir irrigación lubricante, además la placa deja visualizar el momento de la fractura y remover fácilmente las limas rotas.

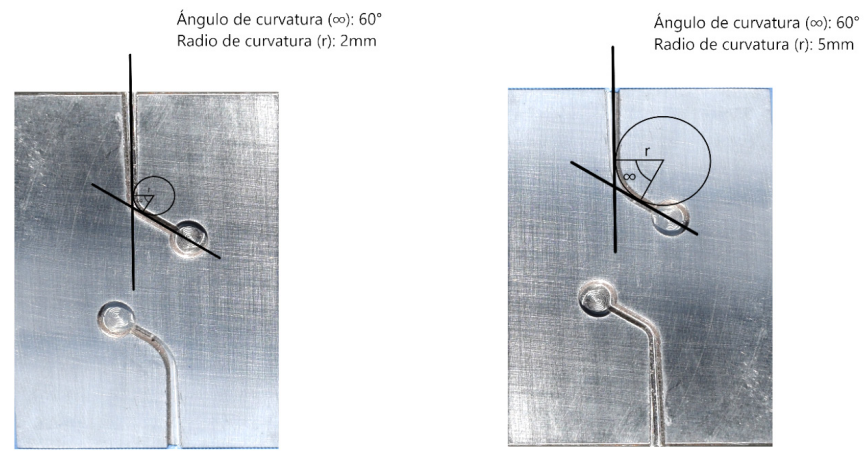

Figura \#2. Bloques de acero inoxidable con 2 canales, con ángulos de curvatura de $60^{\circ}$ y radios de curva de $2 \mathrm{~mm}$ o $5 \mathrm{~mm}$. 


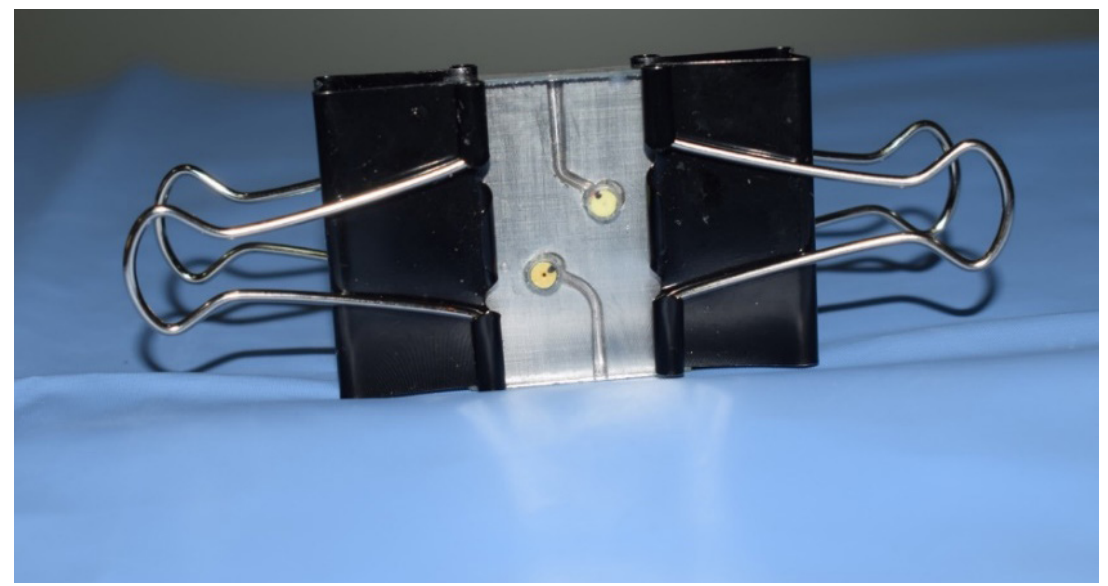

Figura \#3. Fijación con pinzas papeleras para conformar mufla de estudio.

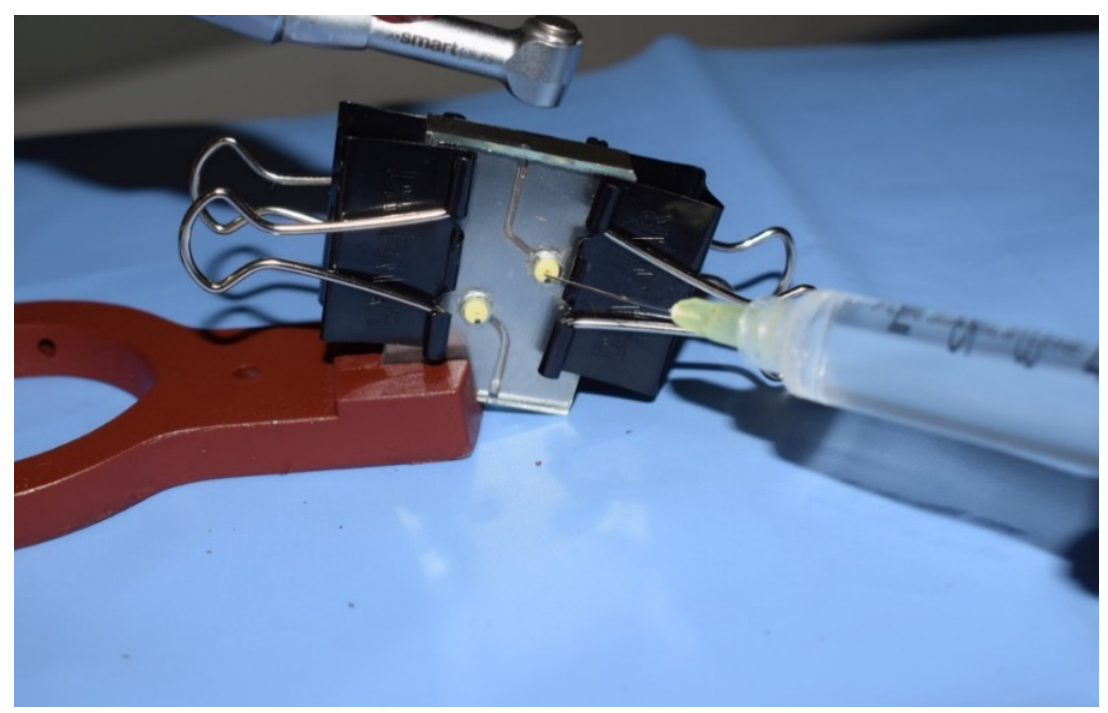

Figura \#4. Técnica de irrigación con hipoclorito de sodio al 5,25\%.

La técnica de instrumentación fue corono apical con movimiento reciprocante siguiendo indicaciones de fabricantes a velocidades y torques recomendadas para ambas marcas de limas, que se accionaron antes de su ingreso al canal y con ligera presión se profundizaron en él hasta llegar a la longitud de trabajo, con irrigación constante de hipoclorito de sodio al $5,25 \%$. Cada canal fue instrumentado por dos o tres limas debido a que por tratarse de acero inoxidable hubo mínimo desgaste de la superficie del conducto. No se utilizaron limas manuales durante de la instrumentación. 


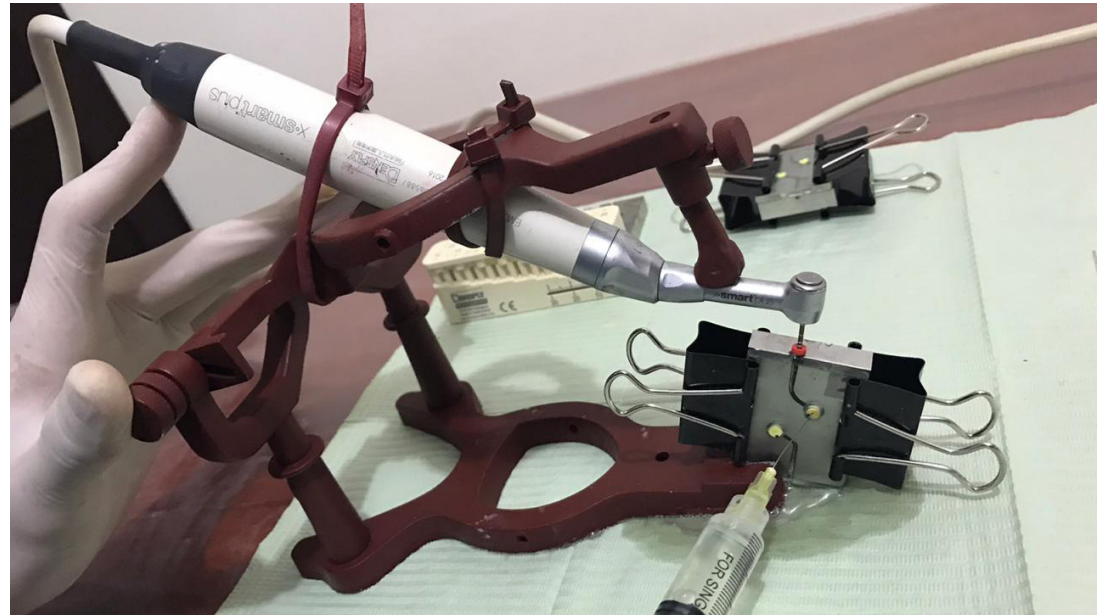

Figura \#5: Instrumentación Lima WaveOne Gold Primary del estudio

La prueba de fatiga cíclica se hizo por el conteo del tiempo en segundos con un cronómetro de "precisión de 0.01 s." desde que se accionó cada lima instrumentando por completo los canales hasta que se presentó la fractura, que pudo ser identificada visual y en algunos casos auditivamente. Mediante la medición del tiempo de fractura se puede calcular el número de ciclos hasta la fractura (NCF) aplicando la fórmula, propuesta por Mohammad. ${ }^{2}$ Que multiplica el tiempo de fractura en segundos (TF) por la velocidad de trabajo con revoluciones por minuto (RPM), tomando en cuenta que WaveOne Gold Primary trabaja con 350 rpm y Reciproc blue R25 con 300 rpm.

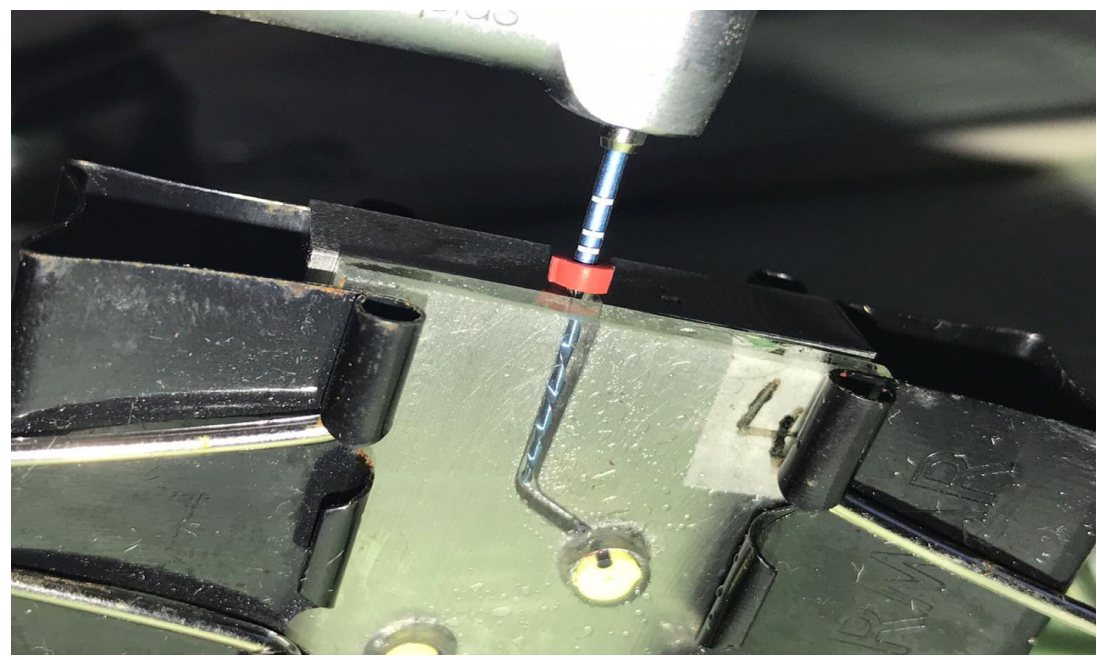

Figura \#6: Visualización de Fractura Lima Reciproc Blue R25 por fatiga cíclica. 
Posteriormente los 20 instrumentos fracturados se llevaron al microscopio de Endodoncia con aumento de 20x para tomar imágenes y observar las superficies de la fractura.

Figura \#7: Imagen 20x de Fractura cíclica de Lima Reciproc R25 del Grupo №4 del estudio.

\section{Resultados}

Los valores obtenidos del número de ciclos hasta la fractura se analizaron estadísticamente con pruebas paramétricas de ANOVA, post hoc Tukey y t de Student. Según la prueba de ANOVA, los valores de medias y desviaciones estándar de la cantidad de números de ciclos hasta la fractura (NCF) se indican en la tabla \#2. Con un nivel de confianza del $95 \%$.

\section{Tabla \#2: Prueba de ANOVA}

\begin{tabular}{|c|c|c|c|c|c|c|c|c|}
\hline \multicolumn{9}{|c|}{ ANOVA } \\
\hline \multicolumn{9}{|c|}{ Tiempos * Revoluciones= NCF } \\
\hline & \multirow[b]{2}{*}{$\mathbf{N}$} & \multirow[b]{2}{*}{ Media } & \multirow[b]{2}{*}{$\begin{array}{c}\text { Desviación } \\
\text { estándar }\end{array}$} & \multicolumn{2}{|c|}{$\begin{array}{c}95 \% \text { del intervalo } \\
\text { de confianza para } \\
\text { la media }\end{array}$} & \multirow[b]{2}{*}{ Minimo } & \multirow[b]{2}{*}{ Máximo } & \multirow[b]{2}{*}{$\begin{array}{l}\text { ANOVA } \\
(p=)\end{array}$} \\
\hline & & & & $\begin{array}{l}\text { Límite } \\
\text { inferior }\end{array}$ & $\begin{array}{l}\text { Límite } \\
\text { superior }\end{array}$ & & & \\
\hline $\begin{array}{c}\text { GRUPO 1: WOG } \\
\text { Py } 2 \mathrm{~mm}\end{array}$ & 5 & 1645,60 & 444,35 & 1093,86 & 2197,33 & 1060,09 & 2082,91 & \multirow{5}{*}{0,000} \\
\hline $\begin{array}{l}\text { GRUPO 2: } \\
\text { WOG P y } 5 \mathrm{~mm}\end{array}$ & 5 & 1704,96 & 315,24 & 1313,54 & 2096,38 & 1308,13 & 2040,33 & \\
\hline $\begin{array}{l}\text { GRUPO 3: RB } \\
\text { R25 y } 2 \mathrm{~mm}\end{array}$ & 5 & 2370,41 & 524,23 & 1719,49 & 3021,33 & 1663,85 & 2996,60 & \\
\hline $\begin{array}{l}\text { GRUPO 4: RB } \\
\text { R25 y 5mm }\end{array}$ & 5 & 3339,96 & 601,88 & 2592,63 & 4087,29 & 2657,20 & 4205,45 & \\
\hline Total & 20 & 2265,23 & 828,93 & 1877,28 & 2653,18 & 1060,09 & 4205,45 & \\
\hline
\end{tabular}


Los resultados fueron que el número de ciclos hasta la fractura (NCF) de dos grupos de estudio el \# 4 y \#3, donde se usan limas Reciproc Blue R25, fue mayor en relación al NFC de los dos grupos de estudio \#2 y \#1 en los que se usa la lima WaveOne Gold Primary. Y en la resistencia a la fatiga cíclica, establecida por el número de ciclos hasta el fallo multiplicado por las revoluciones, el grupo \#4 de la lima Reciproc Blue R25 instrumentada en un canal con un radio de $5 \mathrm{~mm}$ fue significativamente superior que la lima del mismo sistema instrumentada en un canal con radio de $2 \mathrm{~mm}$; y además fue significativamente mayor a la lima WaveOne Gold Primary instrumentada en canales tanto de $2 \mathrm{~mm}$ como de $5 \mathrm{~mm}$ de radio.

\section{Tabla \#3: Prueba de comparaciones múltiples HSD Tukey}

\begin{tabular}{|c|c|c|c|c|c|c|}
\hline \multicolumn{7}{|c|}{ Comparaciones múltiples } \\
\hline $\begin{array}{l}\text { Variable } \\
\text { dependiente: }\end{array}$ & \multicolumn{6}{|c|}{ Tiempos*Revoluciones=NCF } \\
\hline \multicolumn{7}{|l|}{ HSD Tukey } \\
\hline \multirow[t]{2}{*}{ (I)GRUPOS } & & \multirow{2}{*}{$\begin{array}{c}\text { Diferencia } \\
\text { de medias } \\
(1-J)\end{array}$} & \multirow[t]{2}{*}{$\begin{array}{l}\text { Desv. } \\
\text { Error }\end{array}$} & \multirow{2}{*}{$\begin{array}{l}\text { Sig. }(p=) \\
\text { Limite } \\
\text { inferior }\end{array}$} & \multicolumn{2}{|c|}{$\begin{array}{c}\text { Intervalo de } \\
\text { confianza al } 95 \% \\
\end{array}$} \\
\hline & & & & & $\begin{array}{l}\text { Limite } \\
\text { superior }\end{array}$ & \\
\hline \multirow{3}{*}{$\begin{array}{l}\text { GRUPO 1: } \\
\text { WOGPy } \\
2 \mathrm{~mm}\end{array}$} & GRUPO 2: WOGP y $5 \mathrm{~mm}$ & $-59,36$ & 305,60 & 0,997 & $-933,68$ & 814,96 \\
\hline & GRUP0 3: RB R25 y $2 \mathrm{~mm}$ & $-724,81$ & 305,60 & 0,123 & $-1599,14$ & 149,51 \\
\hline & GRUP0 4: RB R25 y $5 \mathrm{~mm}$ & $-1694,36$ & 305,60 & 0,000 & $-2568,69$ & $-820,04$ \\
\hline \multirow{3}{*}{$\begin{array}{l}\text { GRUPO 2: } \\
\text { WOGP y } \\
5 \mathrm{~mm}\end{array}$} & GRUPO 1: WOGP y $2 \mathrm{~mm}$ & 59,36 & 305,60 & 0,997 & $-814,96$ & 933,68 \\
\hline & GRUP0 3: RB R25 y $2 \mathrm{~mm}$ & $-665,45$ & 305,60 & 0,172 & $-1539,77$ & 208,87 \\
\hline & GRUP0 4: RB R25 y $5 \mathrm{~mm}$ & $-1635,00$ & 305,60 & 0,000 & $-2509,32$ & $-760,68$ \\
\hline \multirow{3}{*}{$\begin{array}{l}\text { GRUPO 3: } \\
\text { RB R25 y } \\
2 \mathrm{~mm}\end{array}$} & GRUPO 1: WOGP y $2 \mathrm{~mm}$ & 724,81 & 305,60 & 0,123 & $-149,51$ & 1599,14 \\
\hline & GRUPO 2: WOGP y $5 \mathrm{~mm}$ & 665,45 & 305,60 & 0,172 & $-208,87$ & 1539,77 \\
\hline & GRUPO 4: RB R25 y $5 \mathrm{~mm}$ & $-969,55$ & 305,60 & 0,027 & $-1843,87$ & $-95,23$ \\
\hline \multirow{3}{*}{$\begin{array}{l}\text { GRUPO 4: } \\
\text { RB R25 y } \\
5 \mathrm{~mm}\end{array}$} & GRUPO 1: WOGP y 2mm & 1694,36 & 305,60 & 0,000 & 820,04 & 2568,69 \\
\hline & GRUPO 2: WOGP y $5 \mathrm{~mm}$ & 1635,00 & 305,60 & 0,000 & 760,68 & 2509,32 \\
\hline & GRUPO 3: RB R25 y $2 \mathrm{~mm}$ & 969,55 & 305,60 & 0,027 & 95,23 & 1843,87 \\
\hline
\end{tabular}

Como se observa en la prueba post hoc HSD Tukey, se formaron dos subconjuntos diferentes, uno en donde se tienen los menores valores con los GRUPOS 1, 2 y 3 . Y otro con los mayores valores en donde está el GRUPO 4. Sin embargo para verificar si existe o no diferencias entre estos grupos sin la influencia de los otros, se realiza la prueba t Student 
Tabla \#4: Prueba t Student

\begin{tabular}{|c|c|c|c|c|}
\hline \multicolumn{5}{|c|}{ Prueba T Student } \\
\hline GRUPOS & $\mathbf{N}$ & Media & $\begin{array}{c}\text { Desviación } \\
\text { Estándar }\end{array}$ & $\begin{array}{c}\text { T student } \\
(p=)\end{array}$ \\
\hline GRUPO 1: WOG P y $2 \mathrm{~mm}$ & 5 & 1645,60 & 444,35 & \multirow{2}{*}{0,814} \\
\hline GRUPO 2: WOG P y $5 \mathrm{~mm}$ & 5 & 1704,96 & 315,24 & \\
\hline GRUPO 1: WOG P y $2 \mathrm{~mm}$ & 5 & 1645,60 & 444,35 & \multirow{2}{*}{0,046} \\
\hline GRUPO 3: RB R25 y 2mm & 5 & 2370,41 & 524,23 & \\
\hline GRUPO 1: WOG P y $2 \mathrm{~mm}$ & 5 & 1645,60 & 444,35 & \multirow{2}{*}{0,001} \\
\hline GRUPO 4: RB R25 y $5 \mathrm{~mm}$ & 5 & 3339,96 & 601,88 & \\
\hline GRUPO 2: WOG P y $5 \mathrm{~mm}$ & 5 & 1704,96 & 315,24 & \multirow{2}{*}{0,041} \\
\hline GRUP0 3: RB R25 y 2mm & 5 & 2370,41 & 524,23 & \\
\hline GRUPO 2: WOG P y $5 \mathrm{~mm}$ & 5 & 1704,96 & 315,24 & \multirow{2}{*}{0,001} \\
\hline GRUPO 4: RB R25 y $5 \mathrm{~mm}$ & 5 & 3339,96 & 601,88 & \\
\hline GRUPO 3: RB R25 y 2mm & 5 & 2370,41 & 524,23 & \multirow{2}{*}{0,026} \\
\hline GRUPO 4: RB R25 y $5 \mathrm{~mm}$ & 5 & 3339,96 & 601,88 & \\
\hline
\end{tabular}

En la prueba t Student se hace la comparación entre dos muestras sin tomar en cuenta la influencia de los otros. Y se encontró que sólo son similares $(p>0,05)$ el GRUPO 1: WaveOne Gold Primary y $2 \mathrm{~mm}$ de radio, y el GRUPO 2: WaveOne Gold Primary y $5 \mathrm{~mm}$ de radio.

\section{Discusión}

La dificultad más compleja de la instrumentación es la separación de los instrumentos dentro de los canales radiculares, y como se describe en varios estudios la principal causa es la fatiga cíclica en ellos. ${ }^{34}$ Varios factores influyen para la fractura cíclica como la anatomía radicular compleja con curvas severas, las características físicas y metalúrgicas de las limas, y la técnica o cinemática empleada en la instrumentación. ${ }^{5,6}$

En relación a la anatomía compleja existen varias técnicas para determinar qué tan cerrada, abrupta o severa es la curva de un conducto, y el método de medición del ángulo de curvatura establecido por Schneider en 1971 es el más sencillo y práctico. En muchas investigaciones se ha concluido la incidencia del ángulo de curvatura mayor a $30^{\circ}$ sobre la fractura cíclica de instrumentos rotatorios tratados térmicamente. ${ }^{7.8}$ Sin embargo, la severidad de la curva no solo se establece por el ángulo, es necesario medir el radio de la curvatura, que es en milímetros 
la mitad de la circunferencia tangente a la curva y también se puede calcular por algunos métodos que indican que una curva es más complicada cuando el radio es de menor valor. Debido a que existe escasa evidencia sobre la influencia de los radios de curvaturas en la fatiga cíclica de limas actuales, fue interés de la presente investigación, poner al límite de trabajo a 2 tipos de limas reciprocantes como WaveOneGold Primary y Reciproc Blue R25 cuantificando sus tiempos de fractura cíclica en instrumentación de canales artificiales de acero inoxidable fabricados con $60^{\circ}$ de ángulo y radios de $2 \mathrm{~mm}$ y $5 \mathrm{~mm}$ que corresponden según varios autores a curvaturas severas. ${ }^{61,9,10}$

De igual manera, con el fin generar tensión límite a los instrumentos reciprocantes del estudio in vitro, se eligió el acero inoxidable \#304 para la confección de los conductos artificiales por ser un material de mayor dureza que la dentina. ${ }^{17,18,19} \mathrm{Y}$ es más objetivo utilizar la misma aleación metálica in vitro en lugar de dientes extraídos con tantas variaciones en dureza y valores de curvatura. ${ }^{20}$

Kiefner en el 2013 compara la resistencia a la fatiga cíclica de 2 sistemas Reciproc y Mtwo en canales artificiales de acero inoxidable, con 60 de ángulo de curvatura y $5 \mathrm{~mm}$ de radio, usando un posicionador para pieza de mano de manera que se logró una técnica de instrumentación objetiva e iqualitaria para ambos sistemas. ${ }^{11}$ Inspirados en esta metodología se confeccionó de similares características un soporte de la pieza de mano con un articulador odontológico modificado que estabiliza los bloques de acero con los canales artificiales y las limas para una instrumentación estandarizada.

El movimiento reciprocante es la cinemática más usada ya que los instrumentos tienen un menor contacto o fricción con las paredes internas del canal radicular y así disminuir la tensión sobre ellos. ${ }^{11,12}$ Es considerado por varios autores un movimiento más seguro a la prevención de la fractura que el movimiento de rotación continua. ${ }^{11}$ Lo que nos llevó a utilizar esta cinemática en la actual investigación comparando dos sistemas reciprocantes pero de casas comerciales diferentes Dentsply y VDW.

La prueba de fatiga cíclica se realizó cuantificando con un cronómetro los segundos que tardaba cada una de las limas en fracturarse. Estos valores se multiplicaron por las revoluciones por minutos para obtener el número de ciclos hasta la fractura (NCF), gracias a la fórmula de Mohammad: NCF= rpm (revoluciones por minuto) $\times$ TF (tiempo de fractura). ${ }^{2}$

Los resultados del presente estudio revelaron que la lima Reciproc Blue R25 tuvo mayores NCF que la lima WaveOne Gold Primary; y que cuando la lima Reciproc Blue R25 instrumentó un canal con un radio de $5 \mathrm{~mm}$ demostró mayor resistencia a la fatiga cíclica que cuando instrumentó un canal con radio de $2 \mathrm{~mm}$; y es significativamente más resistente a fatiga cíclica que la lima WaveOne Gold Primary aplicada en canales tanto de $2 \mathrm{~mm}$ como de $5 \mathrm{~mm}$ de radio.

Coincidiendo con el estudio de Cangül Keskin del 2017, sobre "Resistencia de fatiga cíclica de instrumentos reciprocantes", en el cual se instrumentaron canales 
artificiales de acero inoxidable de 60ำ de ángulo de curvatura y $5 \mathrm{~mm}$ de radio, y los resultados fueron que la lima Reciproc Blue R25 tuvo significativamente mayor resistencia a fatiga cíclica que la lima WaveOneGold Primary seguido de la lima Reciproc R25; y analizándolo las limas R25 Blue y R25 tienen igual cinemática, diseño, tamaño y sección transversal en forma de "s", pero difieren solo en sus aleaciones "Blue Wire y M-Wire". ${ }^{29}$

Al comparar las aleaciones del estudio. La "Gold" del sistema WaveOne Gold (Dentsply Maillefer, Suiza) se elabora a través de un procedimiento térmico de la aleación NiTi, sometiéndola a diversas etapas de enfriamiento y calentamiento en diferentes grados de temperatura. El color dorado (oro-gold) se debe a la formación de una capa exterior de Óxido de Titanio. El proceso térmico es posterior al tallado de la lima. ${ }^{13,22}$

La aleación "Blue" del sistema Reciproc Blue es de color azul y más flexible, es hecha con un proceso metalúrgico complejo, al someter el NiTi a extremos cambios térmicos de altas y bajas temperaturas. Se elabora la lima a partir de esta aleación revestida por una capa de óxido debido al tratamiento termo-mecánico. ${ }^{23}$

De acuerdo a los resultados del presente estudio, la lima Reciproc Blue R25 obtuvo mejor tiempo de fatiga hasta la fractura que podría deberse a que su aleación "Blue" se comporta mejor a la prueba de fatiga cíclica que la aleación "Gold" de la lima WaveOneGold Primary. Pero se deben realizar más ensayos de este tipo para corroborarlo.

El tamaño de los dos tipos de limas de la investigación son similares por tener ambas la punta de diámetro \#25 pero diferente conicidad WOG Primary 7\% y Reciproc Blue R25 8\%. 7,15 Sus diseños difieren en la sección transversal, la WaveOne Gold tiene una forma de paralelogramo mientras que la Reciproc Blue es en forma de $S .{ }^{15,24} Y$ aunque hay una falta de acuerdo si la forma transversal del instrumento influye en la resistencia a la fractura, en lo que sí coinciden es en que el área de la sección transversal es causa fundamental de la resistencia a la fatiga cíclica. ${ }^{25,26}$ Por lo que en el estudio actual, se podría atribuir que la lima WaveOne Gold Primary al tener mayor masa en su sección transversal en comparación con la Reciproc Blue de menos masa pudo influir en el resultado de mayor resistencia a la fractura de la Reciproc Blue.

Coincidiendo con el estudio de Gündoğar y Özyürek del 2018, en donde las limas Reciproc Blue R25 mostraron mayor resistencia a fatiga cíclica que las limas WaveOne Gold Primary ya que sus resultados fueron que la lima HyFlex EDM tuvo la mayor resistencia a la fatiga cíclica seguido de la Reciproc Blue R25 y significativamente mayor que la lima WaveOne Gold Primary y la lima OneShape. ${ }^{27}$

Otro factor influyente en los resultados del presente estudio, podría ser el movimiento reciprocante de giros de desplazamiento con diferentes grados para los dos tipos de limas, el sistema WaveOne Gold realiza un primer movimiento cortante $a 170^{\circ}$ en dirección antihoraria y un segundo movimiento de descarga a $50^{\circ}$ en dirección horaria, con una velocidad de 350 rpm . En cambio el sistema Re- 
ciproc Blue tiene un primer desplazamiento cortante antihorario de $150^{\circ}$ y el segundo movimiento horario de 30ํㅡ, a una velocidad de 300 rpm. ${ }^{13,14,15,16}$ Datos que nos hacen pensar que en nuestra investigación las limas Reciproc tal vez tuvieron mejores resultados en resistencia a la fractura cíclica por desplazarse con menos grados antihorario y horario, y por tener menor velocidad de trabajo.

Según los estudios de Özyürek, y otros; Gündoğar \& Özyürek; Keskin, Inan, Demiral, \& Keles; Murilo Priori, y otros; Soram, y otros; en relación al sitio de fractura de los instrumentos dentro de los conductos, todos encontraron que las limas se rompen a nivel del tercio apical. ${ }^{27,28,29}$ Lo que se confirma en esta investigación donde todas las limas Reciproc Blue R25 y WaveOne Gold Primary se fracturaron a nivel apical dentro de los canales de acero.

\section{Conclusión}

La lima Reciproc Blue R25 demostró una mejor resistencia a la fractura, por su mayor número de ciclos antes hasta la fractura, que las limas WaveOne Gold Primary, al instrumentar conductos artificiales con radios de curvatura de $2 \mathrm{~mm}$ y de $5 \mathrm{~mm}$ y con un ángulo de curvatura de $60^{\circ}$. 


\section{Referencias Bibliográficas}

1. Schneider S. W. A comparison of canal preparations in straight and curved root canals. Oral Surgery, Oral Medicine, Oral Pathology. 1971. 271-275.

2. Mohammad I, Khalid M., Mohammed A., Hesham A., Mohammad A., Ali A A., Ebtissam A. M. Comparison of Cyclic Fatigue Resistance of 5 Heat-treated Nickel-titanium Reciprocating Systems in Canals with Single and Double Curvatures. J Endod. 2019. 1-5.

3. Shahabinejad H., Ghassemi A., Pishbin L., Shahravan A. Success of ultrasonic technique in removing fractured rotary nickel-titanium endodontic instruments from root canals and its effect on the required force for root fracture. J Endod. 2013. 824-8.

4. Inan U., Gonulol N. Deformation and fracture of Mtwo rotary nickel-titanium instruments after clinical use. J Endod. 2009. 1396-9.

5. Willershausen B., Kasaj A., Rohrig B. Radiographic investigation of frequency and location of root canal curvatures in human mandibular anterior incisors in vitro. J Endod. 2008. 152-6.

6. Pruett J., Clement D., Carnes D. Cyclic Fatigue Testing of Nickel-Titanium Endodontic Instruments. Journal of endodontics. 1997. 77-85.

7. Adiguzel M., Capar I. Comparison of Cyclic Fatigue Resistance of WaveOne and WaveOne Gold Small, Primary, and Large Instruments. J Endod. 2017. 623-7.

8. De Deus. Blue Thermomechanical Treatment Optimizes Fatigue Resistance and Flexibility of the Reciproc Files. Journal of Endodontics. 2017. 462-466.

9. Lopes H., Elias C., Estrela C., Siqueira J. Assessment of the apical transportation of root canals using the method of the curvature radius. Braz Dent J. 1998. 39-45.

10. Burbano M., Cortés D., Carrillo K., Espinosa E. Radiographic evaluation of the curvature degree and radius in the mesiobuccal canals of maxillary first molars.Odontología.2017.22-32.

11. Kiefner P., Ban M., DeDeus G. Is the reciprocating movement per se able to improve the cyclic fatigue resistance of instruments. International Endodontic Journal. 2013. 430 436.

12. Da Frota M., Espir C., Berbert F., Marques C., Sponchiado-Junior E., Tanomaru-Filho M. Comparison of cyclic fatigue and torsional resistance in reciprocating single-file systems and continuous rotary instrumentation systems. J Oral. 2014. 269-75.

13. Steinfort. Wave One Gold: La nueva apuesta de Dentsply Sirona. Revista de la Sociedad de Endodoncia de Chile Canal Abierto. 2017. 4-7.

14. Vdw-dental [Internet]. VDW GmbH, Munich. Reciproc ${ }^{\circledR}$ blue User Guide. 2016. 1-4. [citado 2020 Dic 09]. Disponible en https://www.vdw-dental.com/en/products/detail/vdwgold-reciproc-blue-24/

15. Yared G. Reciproc blue: the new generation of reciprocation. Giornale Italiano di Endodonzia. 2017. 96-101.

16. Kim H., Kwak S., Cheung G., Ko D., Chung S., Lee W. Cyclic fatigue and torsional resistance of two new nickel-titanium instruments used in reciprocation motion: Reciproc versus WaveOne. J Endod. 2012. 541-4.

17. Slutzky-Goldberg I., Liberman R., Heling I. The Effect of Instrumentation with Two Different File Types, Each with $2.5 \% \mathrm{NaOCl}$ Irrigation on the Microhardness of Root Dentin. Journal of Endodontics. 2002. 311-312. 
18. Cruz-Filho A. M., D. Sousa-Neto M., Saquy P. C., Pecora J. D. Evaluation of the Effect of EDTAC, CDTA, and EGTA on Radicular Dentin Microhardness. Journal of Endodontics. 2001. 183-184.

19. Ibarra Echeverria M., Núñez Solís E., Huerta Ibáñez J. M. Manual ACEROS INOXIDABLES. Tra edición. Santiago de Chile: INDURA. 63.

20. Özyürek T., Gündoğar M., Yılmaz K., Uslu G. Bending resistance and cyclic fatigue life of Reciproc Blue, WaveOne Gold, and Genius files in a double (S-shaped) curved canal. J Dent Res Dent Clin Dent Prospect. 2017. 241-246.

21. Topcuoglu H., Duzgun S., Akti A., Topcuoglu G. Laboratory comparison of cyclic fatigue resistance of WaveOne Gold, Reciproc and WaveOne files in canals with a double curvature. Int Endod J. 2017. 713-7.

22. Yáñez A. Nuevas aleaciones Reciproc ${ }^{\oplus}$ Blue: "Una nueva lima, aún mejor". Revista de la Sociedad de Endodoncia de Chile Canal Abierto. 2017. 2-8.

23. De Deus. Blue Thermomechanical Treatment Optimizes Fatigue Resistance and Flexibility of the Reciproc Files. Journal of Endodontics. 2017. 462-466.

24. Aranguren J., Kuttler S. WaveOne Gold Surfea el conducto radicular con confianza. Dentsply. 2015. 30-35.

25. Pirani C., lacono F., Generali L., Sassatelli P., Nucci C., Lusvarghi L., Prati C. "HyFlex EDM: Superficial features, metallurgical analysis and fatigue resistance of innovative electro discharge machined NiTi rotary instruments. International Endodontic Journal. 2016. 483-93.

26. Mehmet Emin K., Capar I. D., Ertas H. Evaluation of the cyclic fatigue and torsional resistance of novel nickel-titanium rotary files with various alloy properties. Journal of Endodontics. 2016. 1840-43.

27. Gündoğar M., Özyürek T. Cyclic fatigue resistance of OneShape, HyFlex EDM, WaveOne gold, and Reciproc blue nickel-titanium instruments. J Endod. 2018. 1192-6.

28. Özyürek T., Gündoğar M., Uslu G., Yılmaz K., Stafoli S., Grande N., Polimeni A. Cyclic fatigue resistances of Hyfex EDM, WaveOne gold, Reciproc blue and 2shape NiTi rotary fles in diferent artifcial canals. Odontology. 2018. 1 - 6.

29. Keskin C., Inan U., Demiral M., Keles A. Cyclic Fatigue Resistance of Reciproc Blue, Reciproc, and WaveOne Gold Reciprocating Instruments. J Endod. 2017. 1-4. 